\title{
S55c Carbon Steel for Heat Treatment Process with Different Medium in Attenuation Measurement using Ultrasonic Testing
}

\author{
Kharudin Ali $^{1^{*}, \text { Johnny Koh Siaw Paw }^{2} \text {, M.Aizat M.Sulaiman }}{ }^{3}$, Ahmed N. Abdalla ${ }^{4}$, Chong Kok Hen ${ }^{5}$, Tan Jian \\ Ding $^{6}$
}

${ }^{1}$ Institute of Sustainable Energy (ISE), UNITEN, 43000 Selangor, Malaysia

${ }^{2}$ Faculty of Electrical and Automation Engineering Technology, TATIUC, 24000 Terengganu, Malaysia

${ }^{3}$ Faculty of Electronic Information Engineering, Huaiyin Institute of Technology, Huai'an Jiangsu, China

*Corresponding author E-mail: kharudin@tatiuc.edu.my

\begin{abstract}
Ultrasonic testing or commonly known as UT is one of the non-destructive testing technique and widely used in oil and gas industrial inspection. This technique mostly used in defect or crack identification of the pipeline and also used for flaw detection/evaluation, dimensional measurements, and material characterization. This paper presents the effect of heat treatment for S55C carbon steel in attenuation measurement by using ultrasonic testing including annealing, tempering, and quenching process. Seawater and oil are used as a medium of quenching process. The fixed excitation frequency at $4 \mathrm{MHz}$ is used and 0 degrees with double crystal is implemented in this measurement. The thicknesses of blocks used are as the sample from $30 \mathrm{~mm}$ until $80 \mathrm{~mm}$. The result shows that the measurement of material attenuation will be decreased after annealing, tempering and quenching process from $40 \%$ until $99 \%$ compared to before the heat treatment process. The highest attenuation decreasing can be seen on the sample block with the $30 \mathrm{~mm}$ thickness in the heat treatment process.
\end{abstract}

Keywords: Ultrasonic testing; non-destructive testing; annealing; tempering; quenching

\section{Introduction}

Material properties are influenced by variety factors. Microstructure features are known to dictate mechanical properties such as yield strength, hardness, corrosion resistance, failure rate, and others.[1]-[5] Manufacturing processes often induce microstructure changes that affect such mechanical properties and hence the performance of structural components. Nondestructive evaluation of microstructure is a topic that gives a great interest applications in aerospace, railroad, and nuclear industries.

Steel is commonly processed via quenching the high-temperature austenite phase to form martensite, a microstructure that may exhibit high strength and low ductility/toughness. As-quenched martensite is typically tempered to achieve a range of strength and ductility/toughness combined, where strength decreases and ductility/toughness increases with higher tempering temperature and longer tempering time. However, a phenomenon known as tempered martensite embrittlement (TME) produces a decrease in impact toughness at room temperature in the tempering temperatures range from 200 to $400{ }^{\circ} \mathrm{C}$ at tempering time of 1 hour. Tempered martensite embrittlement is also manifested through an increase in ductile to brittle transition temperature (DBTT) [6][7]. Austenite decomposition, cementite precipitation, and cementite coarsening have been identified as mechanisms contributing to TME[8]-[10]. While there are different opinions on the specifics of the underlying mechanisms of TME, generally it is accepted that cementite formation plays a large role in the observed embrittlement. The most commonly adopted cause of TME involves the decomposition of retained austenite to ferrite and cementite during the second stage of tempering. Upon quenching from austenitizing temperatures, martensite is formed and thinned out, interlath austenite is often retained.

During tempering, the retained austenite decomposes to form cementite and ferrite, and this interlath cementite provides preferred crack initiation sites and propagation pathways. Continuously heating and tempering via induction heating have been suggested to refine and disperse carbide particles, as compared to traditionally tempered microstructures. The change in carbide size and distribution allegedly improves the toughness properties in tempering temperatures ranging from approximately 500-700 ${ }^{\circ} \mathrm{C}[11]-[13]$. Both faster heating rates and shorter holding times during tempering have been proposed to improve impact toughness within the explored tempering temperature ranges $\left(500-700{ }^{\circ} \mathrm{C}\right)$; however, limited efforts have been focused on applying short-time tempering for high hardness applications at lower tempering temperatures within the regime of TME[14][15].

Furthermore, inconsistencies in manufacturing processes, one of the other factors, can quickly challenge the validity of the assumption that a small set of sample represents a large batch of material in term of the microstructural properties. For material attenuation, defect or flaw detection applications, nondestructive testing (NDT) techniques were widely implemented. Ultrasonic methods are used reliably in these applications. Additionally, the interaction of the sound wave with the medium provides insight into the material microstructure. Thus, this dissertation will focus on the uses of ultrasonic methods for material characterization. The principles of ultrasonic testing are explained in the following section. 


\section{Relation Work}

Carbon steel is a steel contains $0.12-2.0 \%$ carbon interstitial discretion. Increase of carbon in the steel causes increase the hardness and strength through heat treatment. In contrast, carbon steel is ductile. Weld capability will decrease after heat treatment due to increase in carbon content and will lower the melting point. This process will use high carbon steel which is S55C carbon steel that contains $0.48-0.55 \%$ carbon and is a kind of high-quality carbon structural steel. Annealing is a heat treatment method which is a process of heating and subsequently cooling down in the furnace to produce high durability and ductility but low hardness properties based on microstructure.

\subsection{The Principle of Heat Treatment}

Structural changes to austenite can be achieved by adequate heating based on elastic conditions i.e. to the FCC steel phase and slowly cooled to room temperature. The steel properties from this process are pearlite, which has low strength steel and high ductility. Steel is annealed before being processed by forming a cold, for the reduction of load and energy as well as obtaining a large strain without failure. Tempering is heating of hardened article to temperature below the critical range depends on the hardness to be removed and toughness to be imparted. High-temperature tempering to the part is subjected to high stresses, impact load, and improved high ductility and retains hardness. Quenched steel is usually tempered, by heating the steel to a temperature below the eutectoid temperature for a specific period of time. This gives the steel enough energy to allow the carbon atoms within the martensite to diffuse to lower energy points, forming tempered martensite[16]-[18]. Figure 1 shows the heat treatment graph for S55C carbon steel material.

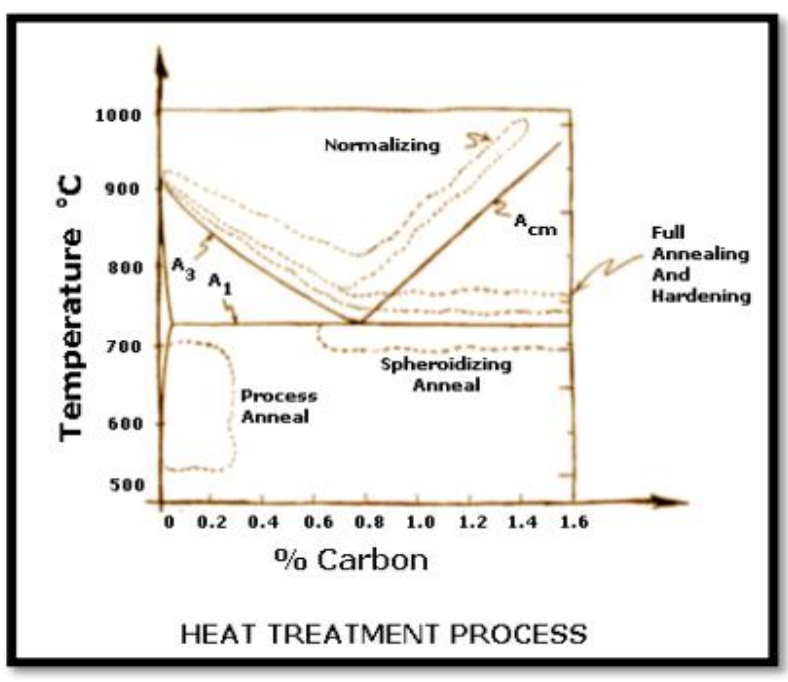

Fig.1: S55C Heat Treatment Process

Ultrasound tests are widely used to detect cracks, hollow leakage entry and also in assessment of material properties [18], [19]. Aiming method and acoustic velocity are used for that purposes. The ultrasonic wave acoustic velocity can be calculated based on the following formulas (1) and (2)[20], [21]:

$\mathrm{VL}=\operatorname{sqr}[(\mathrm{E}(1-\mu)) /(\mathrm{e}(1+\mathrm{u})(1-2 \mu))]$

$\mathrm{VT}=\operatorname{sqr}[\mathrm{E} /(2 \mathrm{e}(1+\mathrm{u}))]$

Where:

E: elastic modulus

P: density

$\mu$ : Poisson's ratio

VL: elastic constants and longitudinal velocity
VT: elastic constants and transverse velocity

Based on the equation above the acoustic velocity is constant across the properties of the material under certain conditions. Therefore, change in the ultrasonic direction is due to changes in the material's morphology[5]. This can be seen based on the formula below. When the ultrasonic wave moves at a certain medium intensity (I) or amplitude (A), it will shorten the exponent to the sound spreading distance (D)[20], [22]. The attenuation coefficient is also a function of detective frequency[23].

$\mathrm{A}=\mathrm{A} 0 \exp (-\mathrm{aD})$ or $\mathrm{I}=\mathrm{I} 0 \exp (-\mathrm{aD})$

Where

A0: Initial sound amplitude

I0: Intensity

a: Attenuation coefficient

Equation 4 shows the affected attenuation coefficient of the ultrasonic wave by absorption and scattering [23]

$a(f)=[20(\log (A 1 / A 2)+2 \log R) / 2 d]$

$a(f): d B / m m$

A1 and A2: peak amplitudes of the first and second transmitted pulses in $\mathrm{mm}$

d: specimen thickness mm

$\mathrm{R}$ : Reflection coefficient

Transmission electron microscopy (TEM), Electron probe microanalysis (EPMA) and Microscopy (SEM) are used for Microstructures to identify the structural phase. Figure 2 shows the heat treatment process by including the temperature range in Kelvin (K) [20].

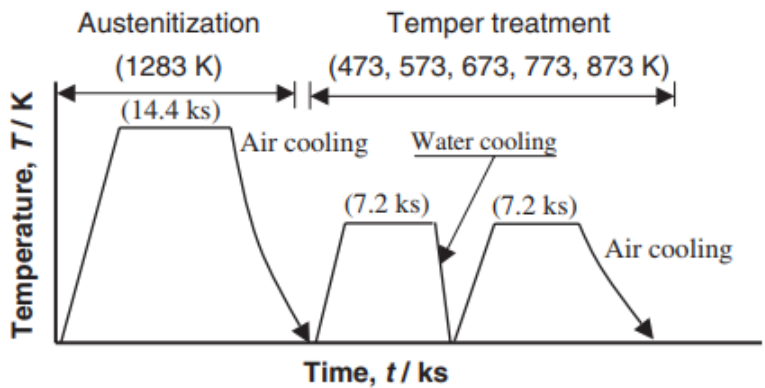

Fig. 2: Heat treatment processing of the experimental material

The microstructure from the observational as-cast material primarily belonged from martensite and ferrite forms in the matrix with $\mathrm{Cr}$ carbide films close to grain limits. SEM and EPMA micrographs from the as-cast sample are demonstrated in Fig. 3(a). It dismissed followed $\mathrm{Cr}$ rich falls close to the martensitic grain limit This carbide cost mostly extinguished along austenitizing at 1283 $\mathrm{K}$ for $14.4 \mathrm{ks}$ and then quenching, as demonstrated in Fig. $3(b)[20]$
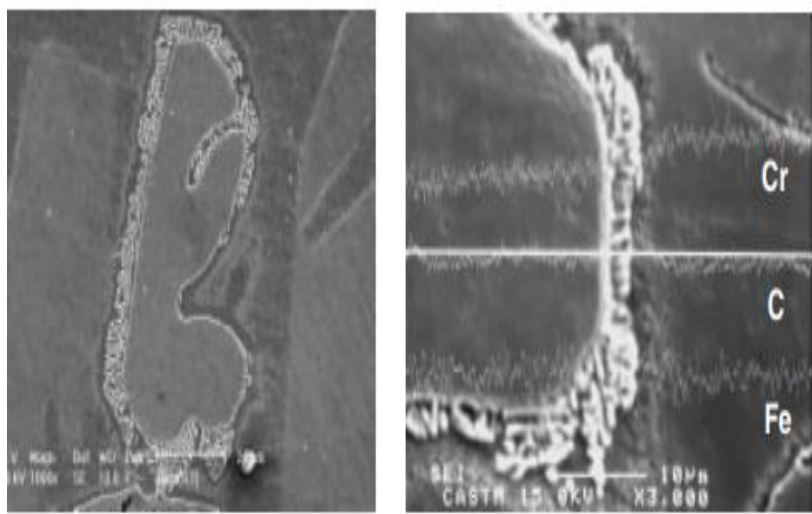

(a) 

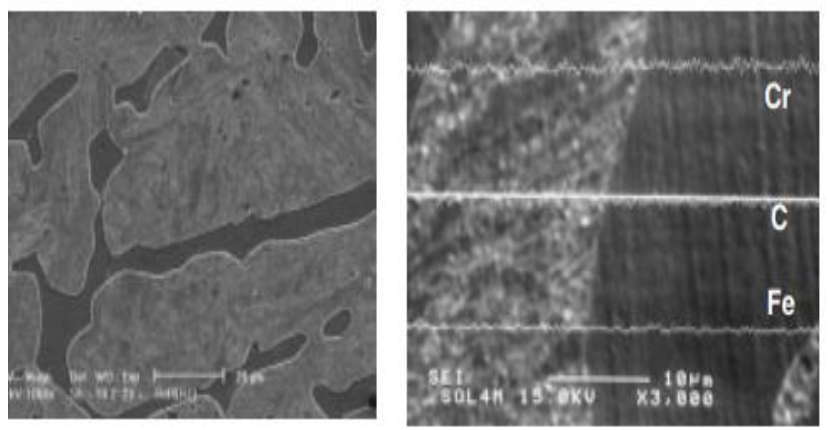

(b)

Fig. 3: SEM and EPMA scan of the experimental material: (a) as-cast, (b) as-quenched

\section{Proposed Method}

Figure 4 shows heat treatment process. First step is preparing carbon steel block into the eight identical samples. Four of the samples size is $70 \mathrm{~mm} \times 50 \mathrm{~mm} \times 30 \mathrm{~mm}$ and others four samples size is $80 \mathrm{~mm} \times 60 \mathrm{~mm} \times 40 \mathrm{~mm}$. All samples will be undergoing different method of heat treatment such as annealing, tempering, quenching in sea water and quenching in oil. The samples will be marked as sample A until H. Sample marked with $\mathrm{C}$ and $\mathrm{F}$ will undergo annealing process, sample $\mathrm{D}$ and $\mathrm{H}$ will undergo the tempering process, sample $A$ and $G$ will undergo quenching process in oil and lastly sample B and $\mathrm{E}$ will undergo quenching process in sea water.

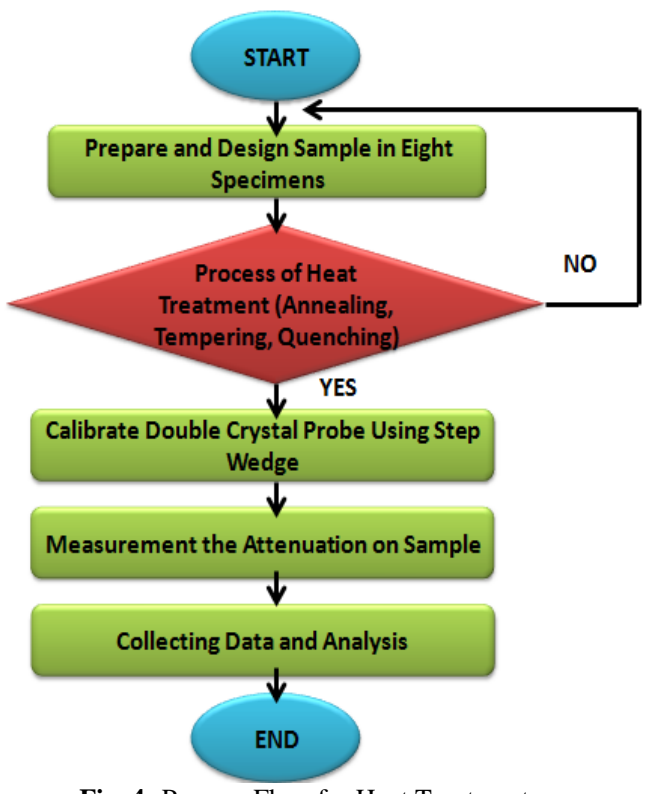

Fig. 4: Process Flow for Heat Treatment

\subsection{Process of Heat Treatment}

Annealing is a heat treatment process that involves heating the steel and then cooling the steel slowly at room temperature. Before starting the process, the oven of the heat treatment machine needs to be heated for a few minutes. The sample will be transferred to the oven and heated at a specific range of the temperature. In this experiment, the 850-degree Celsius is set for furnace according to the melting point of the sample. Time taken to reach 850 Celsius is around 1 hour. Figure 5 shows the heating process of the samples. The samples will be left in the oven at 850 Celsius for 35 minutes. The time is set based on the thickness of the sample which is $1 \mathrm{~mm}$ for 1 minute. After the heating process is complete the sample will be taking out and left at room temperature. The time taken for cooling the sample takes around 3 hours until it reach 29 Celsius (room temperature).

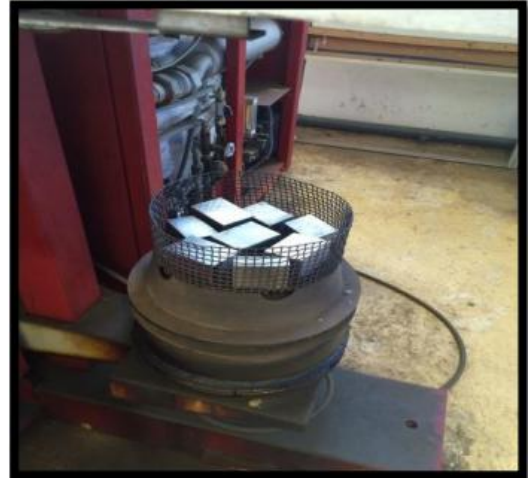

Fig. 5: Annealing Process

Tempering or heating of hardened article to the temperature below the critical range depends upon the hardness to be removed and toughness to be imparted in the process. The effect of hightemperature for tempering process will be give the impact to high stresses, impact load, and improved high ductility and retains hardness. Another effect of tempering is it can improve yield point of structural steels (pearlite class) which have already been hardened by quenching. Tempering is heating the material that has been quenched and hardened for in adequate period of time. Before starting the process, the oven of the heat treatment machine needs to be heated a few minutes. The sample will be put in the oven and heated to a specific range of temperature, which is $850 \mathrm{C}$ in the furnace according to the table of the melting points of the sample. The time taken to reach $850 \mathrm{C}$ is 1 hour. Figure 6 shows process of heating the samples. After reach the $850 \mathrm{C}$, the sample will leave in the oven in 35 minutes before going to the next process.

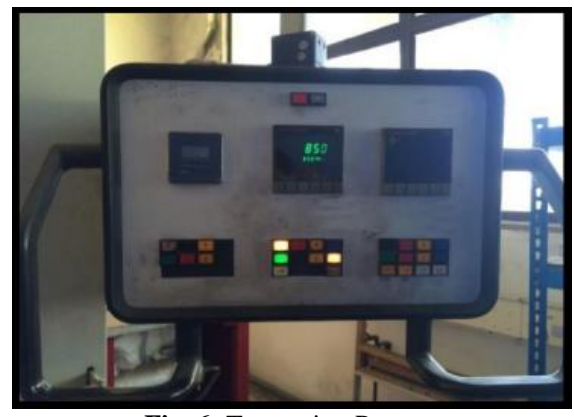

Fig. 6: Tempering Process

After completing the heating process, the sample will be quenched immediately in the water for about 30 minutes until reach to room temperature which is at $29 \mathrm{C}$. The transfer process should take less than one second. Figures 7 shows the samples are quenching in water before reheating. After the sample completely cooled, the sample then will be reheated again in the oven until reaches $850 \mathrm{C}$. The last step is cooling the sample in the air for about an hour until reach room temperature $29 \mathrm{C}$. Time and temperature have important effect of tempering. The process should follow the procedure of the tempering process.

\subsection{Medium of Quenching}

The sample will be heated in the oven of the heat treatment oven. The sample will be held in the oven while heating at $850 \mathrm{C}$ for 35 minutes based on the thickness of the sample. After completing the heating process, the sample will be immediately cooled by quenching in the sea water for 30 minutes until reach room temperature, 29. Figure 7 shows the quenching in sea water. The samples transfer process should take less than one second in the sea water. 


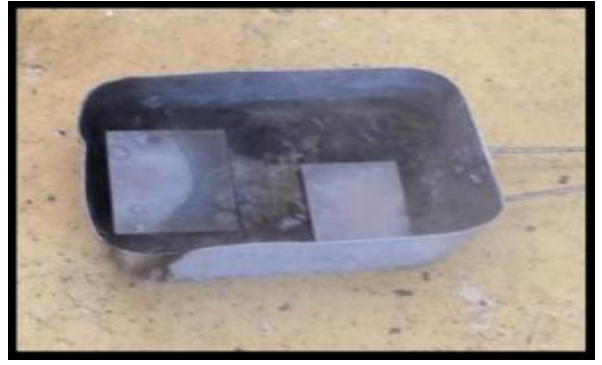

Fig. 7: Quenching in Sea Water Medium

Figure 8 shows the process of quenching in oil. The process is as same as the quenching in sea water but with oil as its medium.

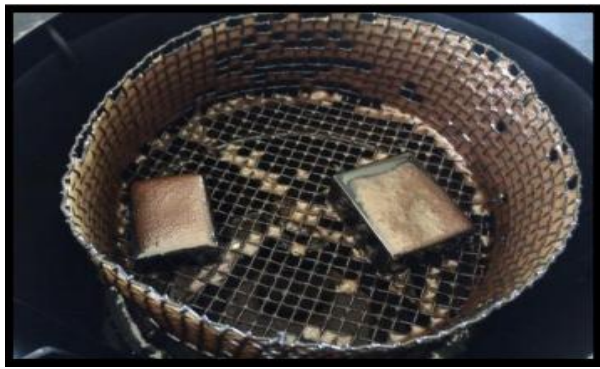

Fig. 8: Quenching in Oil Medium

\section{Experiment Setup}

The scanning process is used for finding the attenuation of carbon steel material. In this process, the Ultrasonic tester is used for measuring the carbon steel S55C sample with different thickness. Ultrasonic Testing is set according to the Table 1 where the specification parameter needs to be set at tester by using 0-degree probes before use it.

Table 1: Specification Set Up Ultrasonic Testing Set

\begin{tabular}{|l|l|}
\hline \multicolumn{2}{|c|}{ Table 1: Specification Set Up Ultrasonic Testing Set } \\
\hline Velocity & VALUE \\
\hline Range & 5960 \\
\hline Probe Delay & $100 \mathrm{~mm}$ \\
\hline Display Delay & $0 \mu \mathrm{s}$ \\
\hline Energy & $0 \mu \mathrm{s}$ \\
\hline Voltage & Low \\
\hline Damping & Low \\
\hline PRF Mode & 100 OHM \\
\hline Frequency & Auto Low $400 \mathrm{~Hz}$ \\
\hline Rectify & $4 \mathrm{MHz}$ \\
\hline Dual & Full Wave \\
\hline & On \\
\hline
\end{tabular}

The 0 probe needs to be calibrated before scanning process is started. The step wedge is used in Calibrate probe process for accuracy data scanning. The steps of calibrating is, first set the $\mathrm{S}$ ref 1 for $5 \mathrm{~mm}$ and S-ref2 for $20 \mathrm{~mm}$ then set full screen high (FSH) and record the first and second echoes. Figure 11 shows the process of calibration by using the 0 -degree probe.

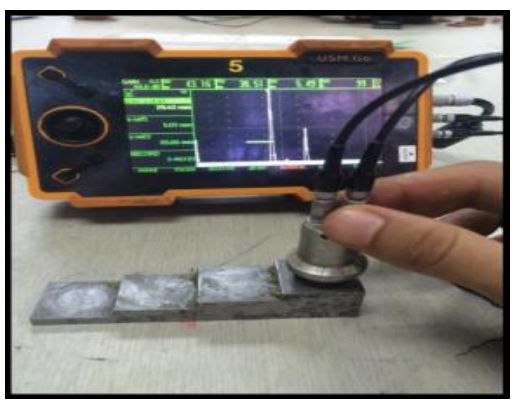

Fig. 9: Calibrate the Probe Process
This experiment will use eight carbon steel blocks. Four samples with dimension $70 \mathrm{~mm} \times 50 \mathrm{~mm} \times 30 \mathrm{~mm}$ and the other four is $80 \mathrm{~mm} \times 60 \mathrm{~mm} \times 40 \mathrm{~mm}$. Figure 10(a) shows the S55C Carbon Steel Sample. All of the samples will be scanned for data record. Before the scanning process is started, grease is applied at the block sample surface to ensure better sound penetration into the samples and at the same time any air that may be present between probe and surface can be excluded. First, the scan is done at different thickness samples for $\mathrm{H} 1$ and $\mathrm{H} 2$ echoes value. The tester is set for $100 \%$ echoes and $\mathrm{H} 1$ and $\mathrm{H} 2$ echoes will be recorded in percents. Figure 10 (b) shows the scanning sample by using the 0 degree probe. The data will be recorded and the process will be repeated in all eight samples with different thickness.

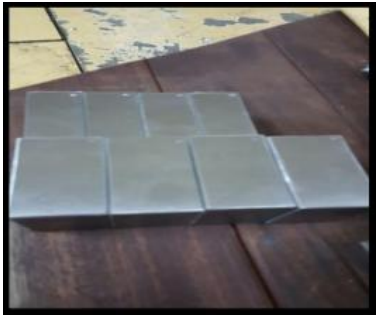

(a)

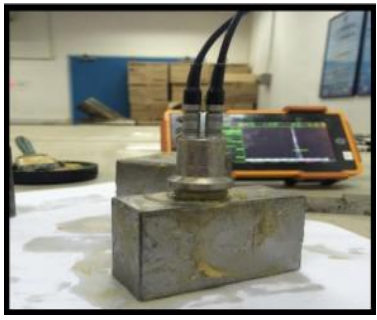

(b)
Fig. 10: Steps for Ultrasonic Testing Scanning (a) S55C Carbon Steel Sample, (b) Scanning the Specimen.

Next, calculate the material attenuation of all specimens by using all data collected. Before that, the attenuation coefficient must be calculated. $\mathrm{H} 1$ and $\mathrm{H} 2$ echoes will be put in the attenuation coefficient to get the value in $(\mathrm{dB})$. The attenuation coefficient is the difference in $\mathrm{dB}$ between $\mathrm{H} 1$ and $\mathrm{H} 2$ echoes. Calculate the attenuation coefficient of all specimens with different thickness based on their $\mathrm{H} 1$ and $\mathrm{H} 2$. Calculate and record all the measurements. The equation (5) shows the equation used for the attenuation coefficient.

Attenuation Coefficient $(\mathrm{dB})=20 \log (\mathrm{h} 1 / \mathrm{h} 2)$

For the last step, calculate the material attenuation. By using the data of the attenuation coefficient the material attenuation can be calculated with its formula. The difference in $\mathrm{dB}$ between $\mathrm{H} 1$ and $\mathrm{H} 2$ echoes will be subtracted by $6 \mathrm{~dB}$ for the natural loss for the carbon steel material then divide with travel distance. Travel distance is determined by the thickness of material multiply by two for second echoes used. Attenuation is measured in $(\mathrm{dB} / \mathrm{mm})$. All data will be collected before and after applying heat treatment and put on the table. The equation (6) shows the formula used to calculate material attenuation.

Material Attenuation $=($ Attenuation Coefficient $-6 \mathrm{~dB}$ (natural loss)) / (thickness x 2)

\section{Result and Discussion}

The result shows that the different amplitude of the signal can be looked at UT set tester before and after the heat treatment process. Sample result for $60 \mathrm{~mm}$ sample thickness in 11(a) before and (b) after heat treatment in the annealing process is shown in Figure 12(a) and 12(b). Here, value of decibel (dB) from ultrasonic probes are lower before heat treatment and based on equation 6 , result for material attenuation will be higher before heat treatment compared to after the heat treatment. The results continue to tempering process within before heat treatment $11(\mathrm{c})$ and $11(\mathrm{~d})$ after heat treatment. The result shown in both processes, the value of the attenuation coefficient and material attenuation are lower before heat treatment. It means that the microstructure changes on the material will be detected on ultrasonic testing technique besides the condition and failure/crack on pipes or plate samples. 


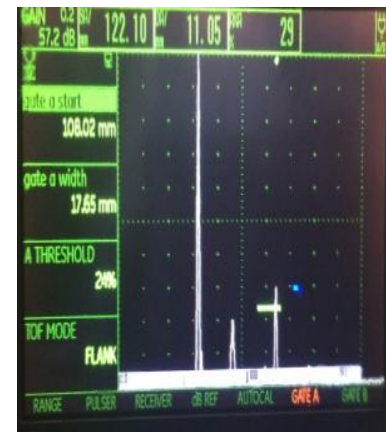

(a)

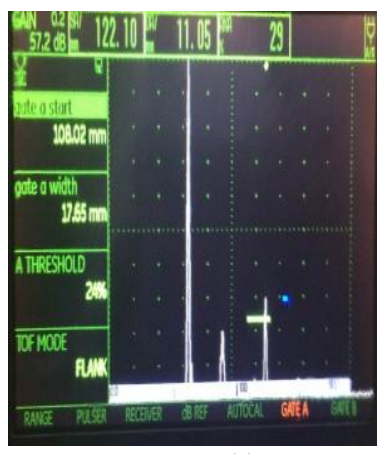

(c)

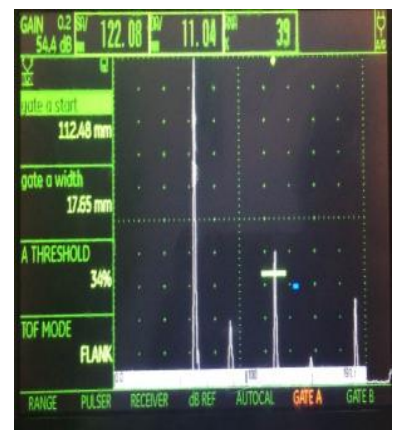

(b)

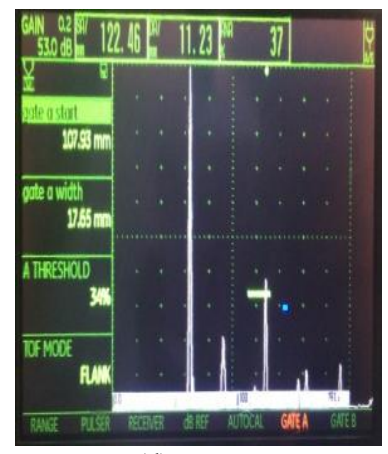

(d)
Fig. 11: Annealing signal and Tempering signal result (a)Annealing before heat treatment, (b) Annealing after heat treatment (a) Tempering before heat treatment, (b) Tempering after heat treatment

In the quenching process, there are two mediums of cooling testing, first, in oil and second in seawater medium. Figure 12 shows quenching result where the sample thickness is $60 \mathrm{~mm}$. Both results showed that values of attenuation coefficient and material attenuation are higher at the beginning process 12(a) and 12(c) compared to after cooling in oil 12(b) and seawater 12(d) medium. But when compare between oil medium and sea water medium being made, the sea water medium shows lower attenuation coefficient and material attenuation value compared to oil medium.

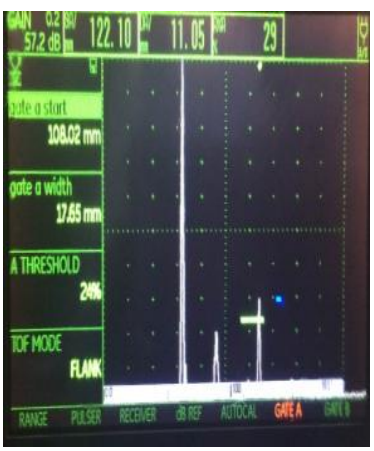

(a)

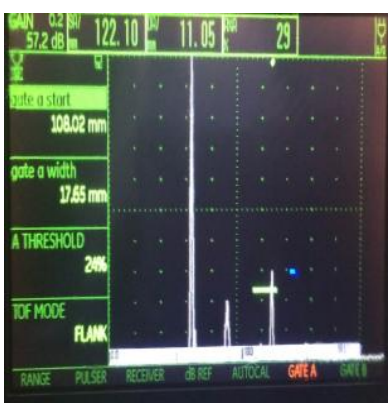

(c)

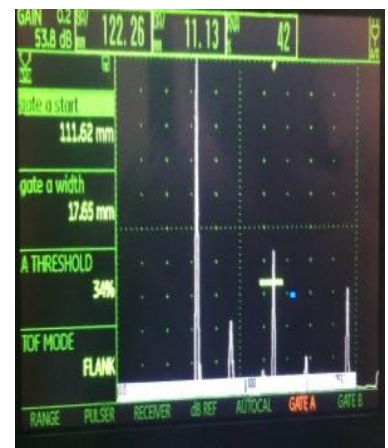

(b)

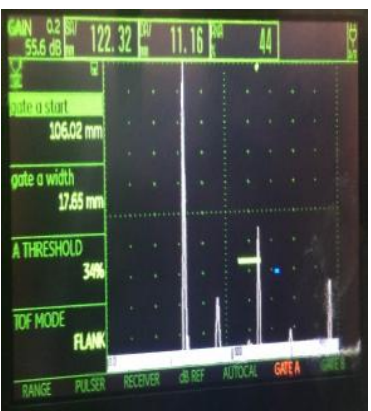

(d)
Fig. 12: Quenching in Oil signal and seawater signal result (a) Quenching in Oil before heat treatment, (b) Quenching in Oil after heat treatment, (c) Quenching in seawater before heat treatment, (b) Quenching in seawater after heat treatment.

\subsection{Material Attenuation before and after Heat Treat- ment}

Table 2 shows comparison material attenuation before and after applying different method of heat treatment. The entire sample with different thickness, $30 \mathrm{~mm}, 40 \mathrm{~mm}, 50 \mathrm{~mm}, 60 \mathrm{~mm}, 70 \mathrm{~mm}$, and $80 \mathrm{~mm}$ are undergoing different process of heat treatment such as annealing, tempering, quenching in oil and sea water. By using all the data collected we can see the difference of material attenuation for all samples before and after applying the different method of heat treatment. From the table, it shows the value of material attenuation for all sample is decrease after applying heat treatment process. From this table, it shows clearly the difference for material attenuation values before and after applying different method of heat treatment according to the respected thickness. For the thickness $30 \mathrm{~mm}$, the attenuation value of quenching process in seawater showed the lowest value which is $0.0004 \mathrm{~dB} / \mathrm{mm}$ whereas tempering process showed the highest attenuation values which is $0.029 \mathrm{~dB} / \mathrm{mm}$ if compare the others. .For the thickness $40 \mathrm{~mm}, 50$ $\mathrm{mm}, 60 \mathrm{~mm}, 70 \mathrm{~mm}$, showed the lowest changes value of attenuation after the heat treatment is also from quenching process in sea water. At thickness of $80 \mathrm{~mm}$, the lowest value of attenuation changes after heat treatment process is from quenching process in oil which is $0.014 \mathrm{~dB} / \mathrm{mm}$ and the highest value is from tempering process, which is $0.020 \mathrm{~dB} / \mathrm{mm}$. From the result, it shows the best process of heat treatment is quenching in seawater because it gives the lowest attenuation value compared to the other processes.

Table 2: Comparison Material Attenuation Before and After Apply Heat Treatment

\begin{tabular}{|c|c|c|c|c|c|}
\hline & \multirow[b]{2}{*}{$\begin{array}{l}\text { Thickness } \\
(\mathrm{mm})\end{array}$} & \multicolumn{2}{|c|}{ Before Heat Treatment } & \multicolumn{2}{|c|}{ After Heat Treatment } \\
\hline & & $\begin{array}{l}\text { Attenuation } \\
\text { Coefficient } \\
\text { (dB) }\end{array}$ & $\begin{array}{l}\text { Material } \\
\text { Attenuation } \\
(\mathrm{dB} / \mathrm{mm})\end{array}$ & $\begin{array}{l}\text { Attenuation } \\
\text { Coefficient } \\
\text { (dB) }\end{array}$ & $\begin{array}{l}\text { Material } \\
\text { Attenuation } \\
(\mathrm{dB} / \mathrm{mm})\end{array}$ \\
\hline \multirow[t]{6}{*}{ Annealing Process } & 30 & 9.37 & 0.056 & 8.179 & 0.006 \\
\hline & 40 & 9.63 & 0.045 & 7.131 & 0.014 \\
\hline & 50 & 10.461 & 0.044 & 7.535 & 0.015 \\
\hline & 60 & 10.751 & 0.04 & 8.179 & 0.018 \\
\hline & 70 & 11.06 & 0.036 & 8.404 & 0.017 \\
\hline & 80 & 11.37 & 0.034 & 8.874 & 0.018 \\
\hline \multirow[t]{6}{*}{ Tempering Process } & 30 & 9.37 & 0.056 & 7.744 & 0.029 \\
\hline & 40 & 9.63 & 0.045 & 7.959 & 0.024 \\
\hline & 50 & 10.17 & 0.042 & 8.404 & 0.024 \\
\hline & 60 & 10.751 & 0.04 & 8.636 & 0.022 \\
\hline & 70 & 11.06 & 0.036 & 9.37 & 0.024 \\
\hline & 80 & 11.37 & 0.034 & 9.63 & 0.02 \\
\hline \multirow[t]{6}{*}{ Quench in oil process } & 30 & 9.37 & 0.056 & 6.375 & 0.006 \\
\hline & 40 & 9.63 & 0.045 & 6.935 & 0.012 \\
\hline & 50 & 10.17 & 0.042 & 7.131 & 0.012 \\
\hline & 60 & 10.751 & 0.04 & 7.535 & 0.013 \\
\hline & 70 & 11.06 & 0.036 & 7.959 & 0.014 \\
\hline & 80 & 11.37 & 0.034 & 8.179 & 0.014 \\
\hline \multirow[t]{6}{*}{ Quench in Sea Water Process } & 30 & 9.37 & 0.056 & 6.021 & 0.0004 \\
\hline & 40 & 9.9 & 0.049 & 6.745 & 0.0093 \\
\hline & 50 & 10.17 & 0.042 & 6.936 & 0.0094 \\
\hline & 60 & 10.751 & 0.04 & 7.131 & 0.0094 \\
\hline & 70 & 11.06 & 0.036 & 7.744 & 0.012 \\
\hline & 80 & 11.7 & 0.036 & 8.404 & 0.015 \\
\hline
\end{tabular}

\subsection{RSM Result}

\subsection{1 before Heat Treatment Process}

Figure 13(a) shows the result of internally studentized residuals versus the normal percentage of probability. Here, there are four points with higher material attenuation, range between 0.49 $\mathrm{dB} / \mathrm{mm}$ until $0.056 \mathrm{~dB} / \mathrm{mm}$, at points $(2.11,97),(0.88,70)$, $(0.88,75)$ and $(0.08,38)$. Lower points are identified at eight points, which are $(0.87,68),(0.30,57),(0.11,53),(0.10,40)$, ($0.23,25),(-0.23,30),(-0.79,20)$ and $(-0.89,15)$. Range for lower material attenuation is 0.034 to 0.041 . The other points are in medium condition for material attenuation. According to Figure 13, (b) parameter that was affecting material attenuation measurement is the thickness of block sample. When thickness of the block sample is low, the material attenuation is high and vice versa. 


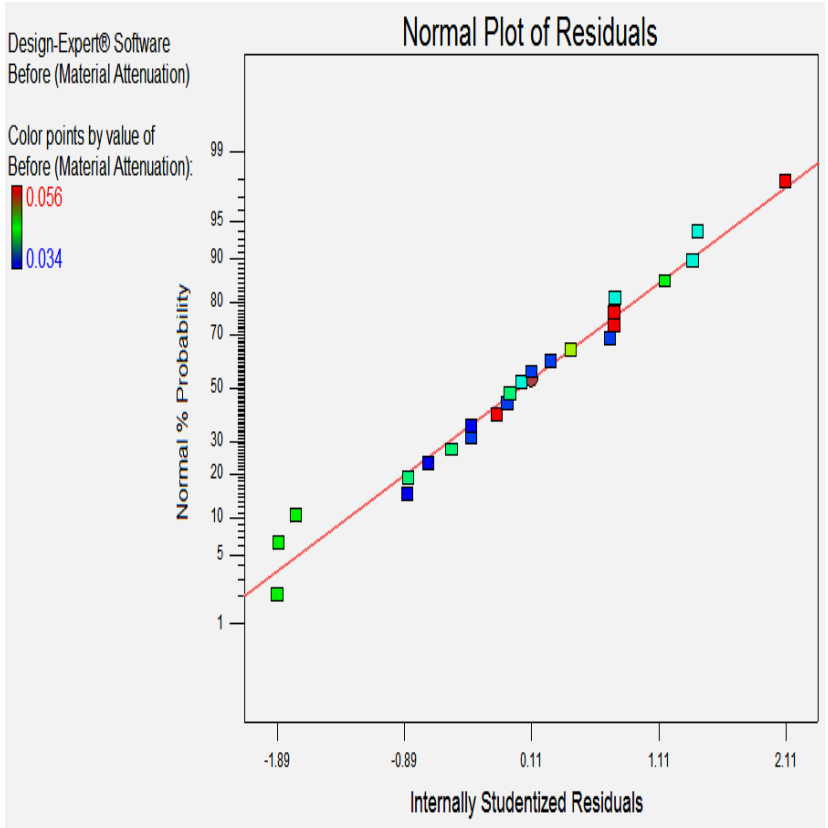

(a)

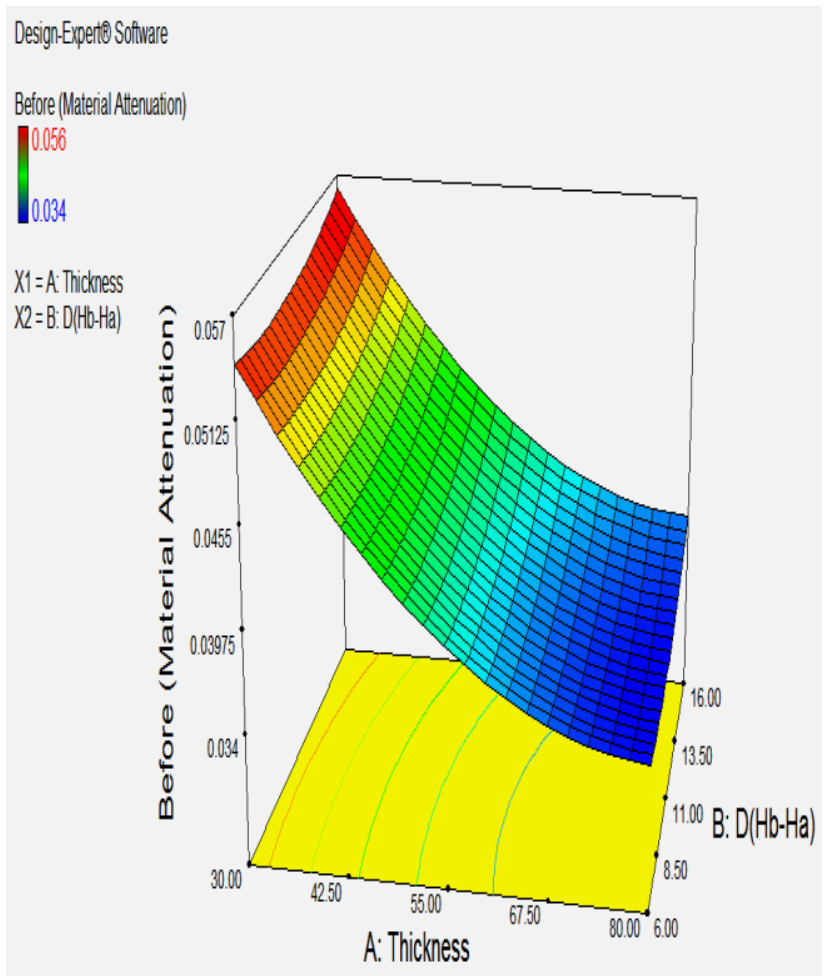

(b)

Fig. 13: RSM result before heat treatment (a) Normal plot versus Residuals (b) Parameter affected the measurement

\subsubsection{After Heat Treatment Process}

From the result on Figure 14(a) shows the material attenuation values $(\mathrm{dB} / \mathrm{mm})$ are decreases unlit $99 \%$. It could be looking on the quantity higher value of material attenuation are decrease until one point at $(1.95,98)$ in the Figure. Then followed by lower material attenuation at two points, which are $(-0.51,25)$ and $(-0.51,28)$. From here effect of heat treatment will decrease the value of material attenuation. According to Figure 14(b) the effect parameter in material attenuation measurement can be concluded as decreasing of the thickness of blocks sample and decreasing the $\Delta$ (Hbefore Hafter) in measurement will increase the material attenuation measurement.

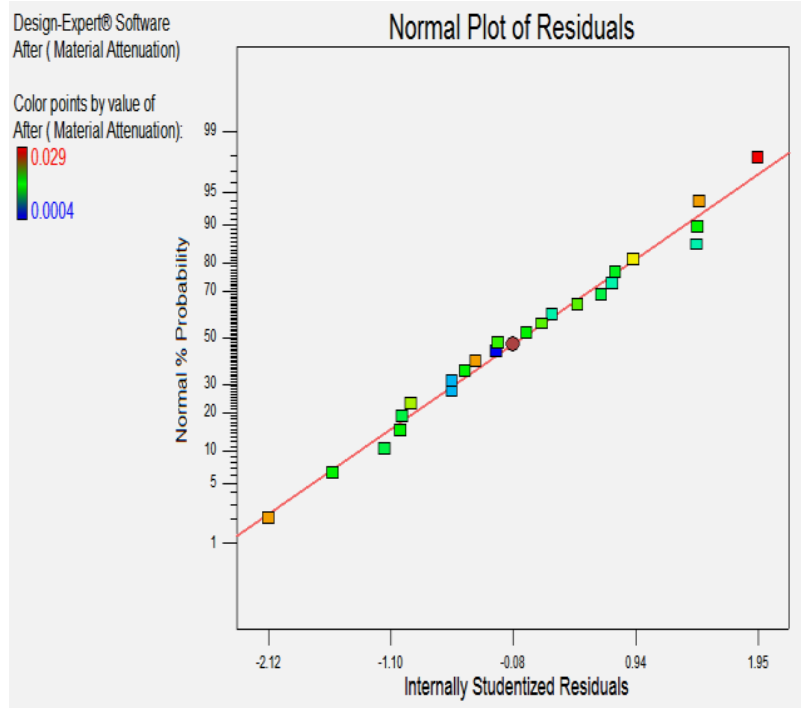

(a)

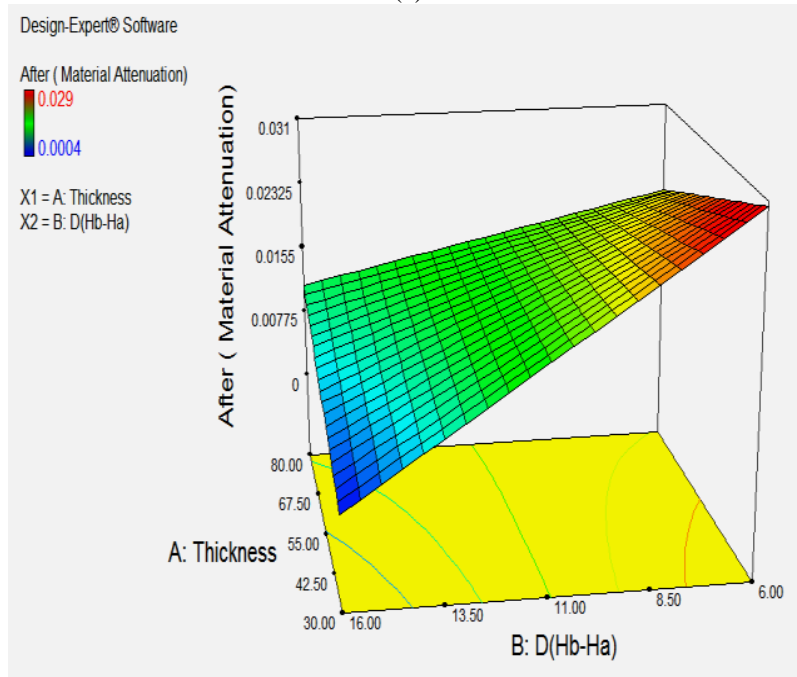

(b)

Fig. 14: RSM result after heat treatment (a) Normal plot versus Residuals, (b) Parameter affected the measurement

\section{Conclusion}

Heat treatment on the carbon steel will effect the changes in material attenuation, before and after the heat treatment. Quench in water process will give the smallest changes value in attenuation loss by using sample block with thickness $30 \mathrm{~mm}$. This due to the sound wave intensity travels through the material much better compared to before heat process. Deduction of material attenuation value showed percentage of the signal after heat treatment increase compared to before the treatment. Quench in seawater showed the lowest attenuation loss and tempering process showed higher attenuation value among all processes. However, all heat treatment processes showed changes and decrease the value of material attenuation. The changes in value were affected by changes in grains structure after the heat treatment process. When the value of the attenuation coefficient is high or large, it means the beam of sound is quickly weakened as it passes through the medium. If the value of the attenuation coefficient is small, it means that the beam of sound easily passing through the medium.

\section{Acknowledgment}

The authors would like to express gratitude to UNITEN for the support of this research under the BOLD scholarship scheme and also under the BOLD funding scheme for Postdoctoral Engineer- 
ing UNITEN also not forget gratitude to TATIUC for facility and equipment for experimental.

\section{References}

[1] S. Gao, A. Shibata, M. Chen, N. Park, and N. Tsuji, "Correlation between Continuous/Discontinuous Yielding and Hall\&amp;ndash;Petch Slope in High Purity Iron," Mater. Trans., vol. 55 , no. 1 , pp. $69-72,2014$

[2] W. Wolf, L. C. R. Aliaga, D. N. Travessa, C. R. M. Afonso, C. Bolfarini, C. S. Kiminami, and W. J. Botta, "Enhancement of Mechanical Properties of Aluminum and 2124 Aluminum Alloy by the Addition of Quasicrystalline Phases," Mater. Res., no. ahead, pp. 0-0, 2016.

[3] N. J. Petch, "The influence of grain boundary carbide and grain size on the cleavage strength and impact transition temperature of steel," Acta Metall., vol. 34, no. 7, pp. 1387-1393, 1986.

[4] K. Bin Ali, A. N. Abdalla, D. Rifai, and M. A. Faraj, "Review on system development in eddy current testing and technique for defect classification and characterization," IET Circuits, Devices Syst., 2017.

[5] A. Abdalla, K. Ali, J. Paw, D. Rifai, and M. Faraj, “A Novel Eddy Current Testing Error Compensation Technique Based on Mamdani-Type Fuzzy Coupled Differential and Absolute Probes," Sensors, vol. 18, no. 7, p. 2108, 2018.

[6] G. Krauss, STEELS: Processing, Structure, and Performance. 2015

[7] D. Rifai, A. N. Abdalla, N. Khamsah, and M. Aizat, "Subsurface Defects Evaluation using Eddy Current Testing," vol. 9, no. March, 2016.

[8] M. Sarikaya, a K. Jhingan, and G. Thomas, "Retained Austenite and Tempered Martensite Embdttlement in Medium Carbon Steels," Metall. Trans., vol. 14, no. June, pp. 1121-1133, 1983.

[9] R. M. Horn and R. O. Ritchie, "Mechanisms of Tempered Martensite Embrittlement in Low Alloy Steels," vol. 9, no. August, 1978.

[10] V. K. Judge, "Effects Of Short-Time Tempering On Mechanical Properties And Fracture Of 4340 Steel," 2017.

[11] C. Revilla, B. López, and J. M. Rodriguez-Ibabe, Carbide size refinement by controlling the heating rate during induction tempering in a low alloy steel, vol. 62. Elsevier Ltd, 2014.

[12] S. Sackl, M. Zuber, H. Clemens, and S. Primig, "Induction Tempering vs Conventional Tempering of a Heat-Treatable Steel," Metall. Mater. Trans. A Phys. Metall. Mater. Sci., vol. 47, no. 7, pp. 3694-3702, 2016.

[13] K. Ali, A. N. A. Alla, M. R. R. M. Amin, D. Rifai, and M. A. Faraj, "AC Excitation Signal Test Frequency for Locating Surface Defect of Heat Treated Brass Calibration Block Using Eddy Current Technique," J. Eng. Apply Sci., 2017.

[14] N. D. Alexopoulos and W. Dietzel, "Effect of corrosion-induced hydrogen embrittlement and its degradation impact on tensile properties and fracture toughness of (Al-Cu-Mg) 2024 alloy," Procedia Struct. Integr., vol. 2, pp. 573-580, 2016.

[15] M. A. Faraj, A. N. Abdalla, F. Bin Samsuri, D. Rifai, and K. Ali, "Investigate of the Effect of Width Defect on Eddy Current Testing Signals under Different Materials," Indian J. Sci. Technol., vol. 10 no. $2,2017$.

[16] J. Adamczyk and a Grajcar, "Heat treatment and mechanical properties of low-carbon steel with dual-phase microstructure," $J$. Achiev. Mater. ..., vol. 22, no. 1, pp. 13-20, 2007.

[17] A. Monden, "Development of Middle-Carbon Steel Bars and Wire Rods for Cold Forging," no. 103, 2013.

[18] A. N. Abdalla, K. Ali, J. Koh, S. Paw, C. K. Hen, and T. J. Ding, "Crack identification using piezoelectric testing on carbon steel pipe for transverse, longitudinal and hole defects with low excitation frequency," vol. 7, pp. 171-176, 2018.

[19] J. K. S. Paw, K. Ali, C. K. Hen, A. N. Abdallah, T. J. Ding, N. A Ahlam, and N.Eirfan3, "Encircling Probe With Multi-Excitation Frequency Signal For Depth Crack Defect In Eddy Current Testing," J. Fundam. Appl. Sci., vol. 10, no. 6S, pp. 949-964, 2018.

[20] C.-H. Hsu, H.-Y. Teng, and S.-C. Chiu, "Ultrasonic Evaluation of Temper-Embrittlement for Martensitic Stainless Steel," Mater. Trans., vol. 44, no. 11, pp. 2363-2368, 2003.

[21] D. Rifai, A. N. Abdalla, K. Ali, and R. Razali, "Giant magnetoresistance sensors: A review on structures and nondestructive eddy current testing applications," Sensors (Switzerland), vol. 16, no. 3, 2016.

[22] B. Ahn and S. S. L. Lee, "Effect of microstructure of low carbon steels on ultrasonic attenuation.," IEEE Trans. Ultrason. Ferroelectr. Freq. Control, vol. 47, no. 3, pp. 620-9, 2000.

[23] L. Slokar, T. Matk, and P. Matković, "Comparison Of Microstructural Characteristics For Biomedical Ti(70,80 At.\%)-CrMo And Ti(70,80 At.\%)-Nb-Zr Alloys," vol. 2, pp. 178-182, 2012. 